\title{
Pandemic (H1N1) 2009 influenza vaccine roll-out in NSW
}

\author{
Jan Fizzell ${ }^{\mathrm{A}, \mathrm{B}}$, Sue Campbell-Lloyd ${ }^{\mathrm{A}}$, Andrew J. \\ Hallett $^{\mathrm{A}}$, Chris P. Lowbridge ${ }^{\mathrm{A}}$, Dennis Meijer ${ }^{\mathrm{A}}$ \\ and Sophie E. Tyner ${ }^{\mathrm{A}}$ \\ A Pandemic (H1N1) Influenza Vaccine Team, NSW Department \\ of Health \\ BCorresponding author. Email: jan.fizzell@doh.health.nsw.gov.au
}

\begin{abstract}
The roll-out of the pandemic (H1N1) 2009 influenza vaccine in NSW was significantly different to that envisaged for a pandemic vaccination program. Pre-pandemic planning had focused on the urgent roll-out of a vaccine through mass vaccination clinics in a time of high demand due to a virulent influenza virus. Instead the situation was less urgent, with the vaccine available only after the peak of incidence of infections in NSW. Consequently mass vaccination clinics were considered to be a less appropriate method of delivering the vaccine than a primary care focused delivery model. This paper describes the program, some of the controversies considered during its roll-out and factors to be considered in planning for future pandemics.
\end{abstract}

A keystone of Australia's pandemic planning has been the introduction of a vaccination program as quickly, safely and effectively as possible after the pandemic strain of influenza is recognised. ${ }^{1}$ Since 2003 the Australian Government has been putting in place sleeper contracts to ensure that when a pandemic strain of influenza emerged Australia would be well-placed to acquire a vaccine.

In April 2009, a novel strain of influenza A (H1N1) was identified. It quickly became apparent that recent seasonal influenza vaccination was unlikely to provide protection against this strain ${ }^{2}$ and a vaccine covering it would be required. On 28 May 2009 the Australian Government placed an order for enough pandemic vaccine to vaccinate approximately 10 million individuals (20 million doses). ${ }^{3}$ This paper describes the pre-pandemic planning for vaccine roll-out, controversies encountered during the roll-out and initial progress of the roll-out in New South Wales (NSW).

\section{Pre-pandemic planning}

NSW pandemic plans, written in preparation for a more severe pandemic, consider the use of mass vaccination clinics an effective method of distributing vaccines to the population. ${ }^{4}$ Consequently guidelines were in development to assist area health services (AHSs) in delivering vaccination clinics. The pandemic vaccine was always likely to be supplied in multi-dose vials due to the increased speed of production. ${ }^{3}$ Needles and syringes to administer a vaccine were stockpiled by the Australian Government.

\section{Progress during the pandemic}

Decisions regarding the supply of the pandemic vaccine were made early in the pandemic, one month after the World Health Organization declared an emergency and well before the disease was established in Australia. It was correctly anticipated that worldwide demand for the pandemic vaccine would outstrip the production capacity of the vaccine manufacturer. This was despite the pandemic being characterised as moderate and the World Health Organization stating that the overwhelming majority of cases recover with no treatment. ${ }^{5}$ By August 2009 it became clear that a vaccine would likely be available only after the first wave of the pandemic had subsided and the rate of admissions for critical care and deaths had decreased.

\section{Controversies in rolling out this program Guillain-Barré syndrome}

In 1976 the United States of America implemented a largescale vaccination program for a swine-origin influenza virus. ${ }^{6}$ Following the roll-out, and particularly in the first 5 weeks after vaccination, a number of people developed Guillain-Barré syndrome (at a rate of around one case per 100000 vaccinated). ${ }^{7}$ This incidence has not been found in other influenza vaccination programs; ${ }^{8}$ nevertheless concerns remained that the $2009 \mathrm{H} 1 \mathrm{~N} 1$ vaccination program might lead to an increase in cases of Guillain-Barré syndrome in the community. In response to these concerns the 


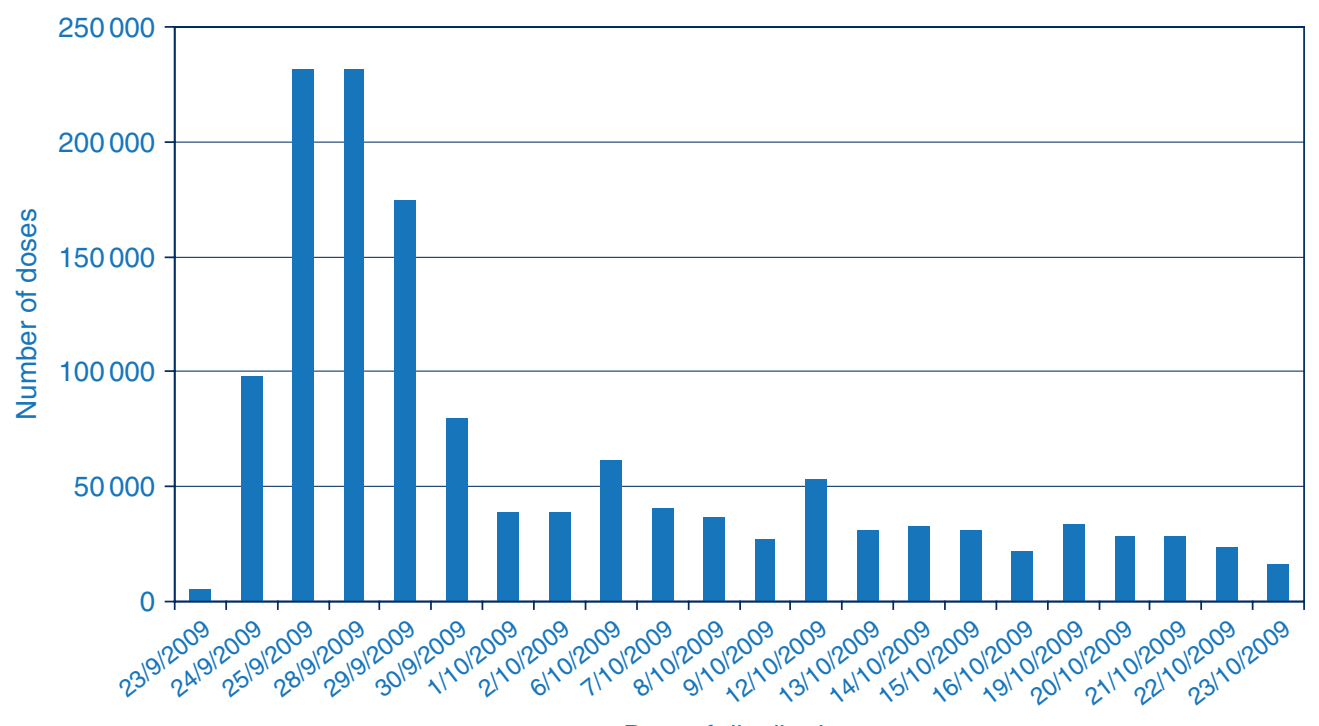

Date of distribution

Figure 1. Doses of pandemic (H1N1) 2009 influenza vaccine distributed in NSW on week days* by date for the period 23 September-23 October, 2009.

*Dispatch does not occur on weekends.

**Authorisation for distribution by the Therapeutic Goods Administration occurred late on 23 September.

Australian Technical Advisory Group on Immunisation released an information sheet about Guillain-Barré syndrome and the vaccine, stating that recently there had not been an increased association between Guillain-Barré syndrome and influenza vaccination and that vaccination may, in fact, be protective against the development of Guillain-Barré syndrome. ${ }^{9}$

\section{Use of a multi-dose vial}

While it has long been documented that multi-dose vials would be used for a pandemic influenza vaccination program, the use of the vials in a mild pandemic elicited concern among health professionals. Several instances of transmission of infectious diseases, from dates prior to the adoption of modern infection control practices, were cited as a cause for concern by infectious disease specialists and subsequently reported in the media. ${ }^{10}$ While it is preferrable to use a singledose pre-filled syringe, due to the potential infection control issues, the worldwide demand for the vaccine was such that taking delivery only in that form would have created a significant delay in vaccine availability.

The inclusion of thiomersal, a preservative containing small amounts of mercury, in the multi-dose vial has also elicited some controversy. While thiomersal has been removed from most childhood vaccines as a precaution during a time when it was theorised that it may cause harmful effects, ${ }^{11}$ the World Health Organization's Global Advisory Committee on Vaccine Safety has concluded that there is '...currently no evidence of mercury toxicity in infants, children, or adults exposed to thiomersal in vaccines'. ${ }^{12}$

\section{Vaccine program development and roll-out in NSW, 2009}

The moderate nature of the pandemic and the fact that a vaccine was only available after the rate of infections was subsiding decreased the need for an urgent roll-out of the vaccine. Within NSW, representative primary care groups and AHSs were consulted regarding preferred methods for vaccine roll-out. Mass vaccination clinics were no longer considered to be appropriate. Rather, a more usual general practioner (GP) and Aboriginal Health Service-based model for delivery of the vaccine was thought to be more accessible and appropriate.

All AHSs developed plans to deliver the vaccine to healthcare workers, to consider opportunistic vaccination of vulnerable patients and to provide assistance in areas where GP and Aboriginal medical services were less accessible.

Once the vaccine was approved, a dedicated webpage with training packages for the safe use of multi-dose vials and a general introduction to the program was uploaded to the NSW Health website. ${ }^{13}$ Links to important national documents and webpages were included.

The program commenced for people aged 10 years and over on 30 September 2009. This involved the distribution of hundreds of thousands of doses of the vaccine $(n=664860)$ 


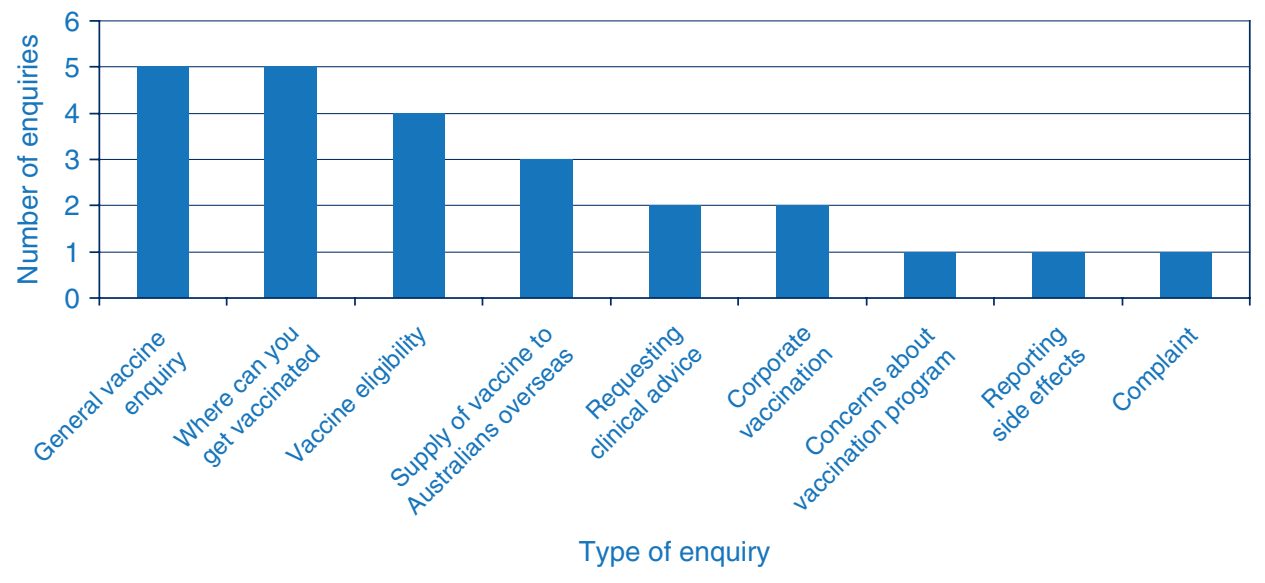

Figure 2. Pandemic (H1N1) 2009 influenza vaccination enquiries to the NSW Health email dropbox for the period 1 September-23 October 2009.

within a 5-day period across NSW. Figure 1 shows the number of doses delivered between 23 September and 23 October 2009.

A number of enquiries (24) were received at the NSW Department of Health's generic email account. The majority were enquiring about gaining access to the vaccine (Figure 2).

A computer-aided telephone interview as part of the NSW Health Survey will monitor vaccine uptake within NSW and NSW Health is working with the Therapeutic Goods Administration to monitor any adverse events following H1N1 vaccination. Triage-text searching of emergency department presentations for persons presenting with possible adverse reactions is also ongoing.

\section{Future planning}

In December 2009 the pandemic vaccine was made available to children aged from 6 months to 10 years. ${ }^{14}$ It has been announced that the 2010 annual influenza vaccination will contain the pandemic strain, leading to a need for clear communications to medical practioners regarding the roles of the pandemic vaccine and the seasonal influenza vaccine. ${ }^{15}$

\section{Pandemics in the future}

For future pandemics, it will be important to consider:

- how best to build confidence around pandemic vaccines so that health professionals are comfortable delivering the vaccination

- how best to communicate to both the health community and the general public the challenges of advancing vaccination program planning with limited available information

- how best to work with the Australian Government and other partners to balance the resources required to plan and manage a pandemic vaccination campaign with those needed by other existing, high-priority health programs and responses.

Future pandemics may be more severe than that caused by the current $\mathrm{H} 1 \mathrm{~N} 1$ virus, resulting in a completely different approach to a national vaccination program. This possibility emphasises the importance of building a degree of flexibility into any pandemic response plan.

\section{Acknowledgments}

The vaccine roll-out could not have occurred without the hard work and co-operation of: the Media and Communications Unit and the Centre for Epidemiology and Research at the NSW Department of Health; the NSW Health area health services; the Primary Care Consultation Group for Pandemic Influenza; and the NSW State Vaccine Distribution Centre.

\section{References}

1. Australian Government Department of Health and Ageing. Part 1 Section 2.4 Protecting the population using vaccination Australian Health Management Plan for Pandemic Influenza December 2008.

2. Centers for Disease Control and Prevention (CDC). Serum cross-reactive antibody response to a novel influenza A (H1N1) virus after vaccination with seasonal influenza vaccine. MMWR Morb Mortal Wkly Rep 2009; 58(19): $521-4$.

3. Australian Government Department of Health and Ageing. Media Release. Available from: http://www.healthemergency. gov.au/internet/healthemergency/publishing.nsf/Content/news039 dated 28 May 2009 (Accessed 23 October 2009.)

4. NSW Health. NSW Health Interim Influenza Pandemic Action Plan. November 2005.

5. World Health Organization. Western Pacific Region Pandemic (H1N1) 2009 - Overview of the current Pandemic H1N1 2009 situation. http://www.wpro.who.int/health_topics/h1n1/ (Accessed 7 December 2009.) 
6. Sencer DJ, Millar JD. Reflections on the 1976 swine flu vaccination program. Emerg Infect Dis [serial on the Internet]. 2006 Jan. Available from: http://www.cdc.gov/ncidod/ EID/vol12no01/05-1007.htm (Accessed 23 October 2009.)

7. Schonberger LB, Bregman DJ, Sullivan-Bolyai JZ, Keenlyside RA, Ziegler DW, Retailliau HF et al. Guillain-Barre syndrome following vaccination in the National Influenza Immunization Program, United States, 1976-1977. Am J Epidemiol 1979; 110(2): 105-23.

8. Haber P, Sejvar J, Mikaeloff Y, DeStefano F. Vaccines and Guillain-Barre syndrome. Drug Saf 2009; 32(4): 309-23. doi:10.2165/00002018-200932040-00005

9. Australian Technical Advisory Group on Immunisation. ATAGI advice regarding influenza, influenza vaccines and Guillain-Barré Syndrome (Dated 7 October 2009.)

10. ABC News online. Swine flu vaccine strategy 'risky'. Available from: http://abc.gov.au/news/stories/2009/ 08/20/2662321.htm (Dated 20 August 2009.)

11. Australian Technical Advisory Group on Immunisation (ATAGI) advice regarding the use of influenza vaccines containing thiomersal. Available at: http://www.healthemergency. gov.au/internet/healthemergency/publishing.nsf/Content/2723 D54E78BF5739CA2576400027E1E9/\$File/ATAGI_Thio statm\%20_091009.pdf (Accessed 22 September 2009.)

12. World Health Organization Global Committee on Vaccine Safety Thiomersal and vaccines: questions and answers http://www.who.int/vaccine_safety/topics/thiomersal/ questions/en/index.html (Accessed July 2006.)

13. NSW Department of Health website. Available from: www.health.nsw.gov.au

14. Therapeutic Goods Administration. Australian Government Media Release: TGA approves Pandemic (H1N1) 2009 influenza vaccine for use in children aged from 6 months of age. http://www.tga.gov.au/alerts/medicines/h1n1vaccine2. htm (Accessed 3 December 2009.)

15. Influenza Specialist Group Influenza Vaccine Composition 2010 accessed online at http://www.influenzaspecialistgroup. org.au/content/view/70/67/ dated 19 October 2009 (Accessed 3 December 2009.) 